\title{
Pyrenocarpous lichens in Goa with five new records to India
}

\author{
Pallavi Randive ${ }^{1}$, Gaurav K. Mishra ${ }^{2}$, Sanjeeva Nayaka ${ }^{2 *}$, D. K. Upreti ${ }^{2}$ \& M. K. Janarthanam ${ }^{1}$ \\ ${ }^{1}$ Department of Botany, Goa University, Taleigao Plateau, Goa 403 206, India \\ ${ }^{2}$ Lichenology Laboratory, CSIR-National Botanical Research Institute, Rana Pratap Marg, Lucknow 226 001, India \\ *Email: nayaka.sanjeeva@gmail.com
}

\section{ARTICLE HISTORY}

Received: 21 May 2021

Accepted: 27 August 2021

Available online: 29 September 2021

\section{KEYWORDS}

Arthopyreniaceae

Biodiversity

Porinaceae

Pyrenulaceae

Taxonomy

Western ghats
ABSTRACT

The pyrenocarpous lichens are the one which produces perithecial ascocarps. They are one of the prominent groups of lichens in tropical forests. Frequent incidence of pyrenocarpous taxa in lichen biota of Goa prompted us to take up the exclusive study of this group in the State. The study revealed the occurrence of 79 species belonging to 15 genera and seven families. The family Pyrenulaceae had the maximum number of 23 species, while 20 belonged to the family Porinaceae. In comparison to North Goa, South Goa is fairly well explored for lichens representing 71 species from 11 localities. The following five species are reported as new to India - Porina exserta, P. siamensis, Pyrenula dissimulans, $P$. pyrenastrospora and $P$. rinodinospora. With the addition of five new records, the Goa State now represents 165 species of lichens, out of which $48 \%$ is represented by pyrenocarpous lichens. The present study will be useful for monographic studies on pyrenocarpous lichens and for environmental monitoring studies in the area, as this can be considered as a key indicator species.

\section{Introduction}

The Western Ghats traverses through the State of Goa, India and is a well-known biodiversity hotspot. Extensive lichen exploration in the State has been initiated recently, and so far, 160 species are reported (1-6). Frequent occurrence of pyrenocarpous lichens during the exploration in the State indicated their dominance which prompted us to carry out an exclusive study on this group. Pyrenocarpous are a group of lichens having perithecia as their ascocarps. Pyrenocarpous taxa commonly grow on the bark of trees, sometimes on rocks, soil or leaves, mostly in moist tropical regions of the world (7).

In the past, pyrenocarpous lichens collected from Goa were included in various monographic works (814) and few new species such as Anthracothecium goaense A. Singh [current name Pyrenula gibberulosa (Vain.) Aptroot] and P. subacutalis Upreti [current name Pyrenula maravalensis Vain.] were also described. The recent floristic studies in the State reported 14 pyrenocarpous taxa from the Cotigao Wildlife Sanctuary (15) and 13 taxa from Bondla and Bhagwan Mahavir Wildlife Sanctuaries (16). A list of lichens from the Goa State reveals 118 species under 42 genera and 23 families, of which 44 were pyrenocarpous lichens (1). Further, in an exclusive survey of Cotigao Wildlife Sanctuary 36 pyrenocarpous taxa were reported under nine genera (3). In the studies on foliicolous lichens, there enumerated a total of 15 pyrenocarpous lichens $(2,4)$. These reports clearly indicate that Goa State has a rich diversity of pyrenocapous lichens. Therefore, carrying out an exclusive study on pyrenocarpous lichens of the State was inevitable. The ultimate aim of the study was to produce a checklist and identification key for pyrenocarpous lichens of Goa State by compilation of previous reports and by more exploration in the State.

\section{Materials and Methods}

The present study is based on published literature, previously collected specimens deposited in the herbarium LWG of CSIR-National Botanical Research Institute, Lucknow and freshly collected specimens from Goa. About 350 specimens from 19 localities of Goa (Fig. 1) were available for the study. Morphological and anatomical characters were examined using stereo zoom Leica S8APO and light DM2500 microscopes attached to a camera. Thin sections of perithecia were cut using a razor blade under a stereo zoom microscope. All anatomical measurements were recorded in plain water, while

(C) Randive et al (2021). This is an open-access article distributed under the terms of the Creative Commons Attribution License, which permits unrestricted use, distribution and reproduction in any medium, provided the original author and source are credited (https://creativecommons.org/licenses/by/4.0/). 


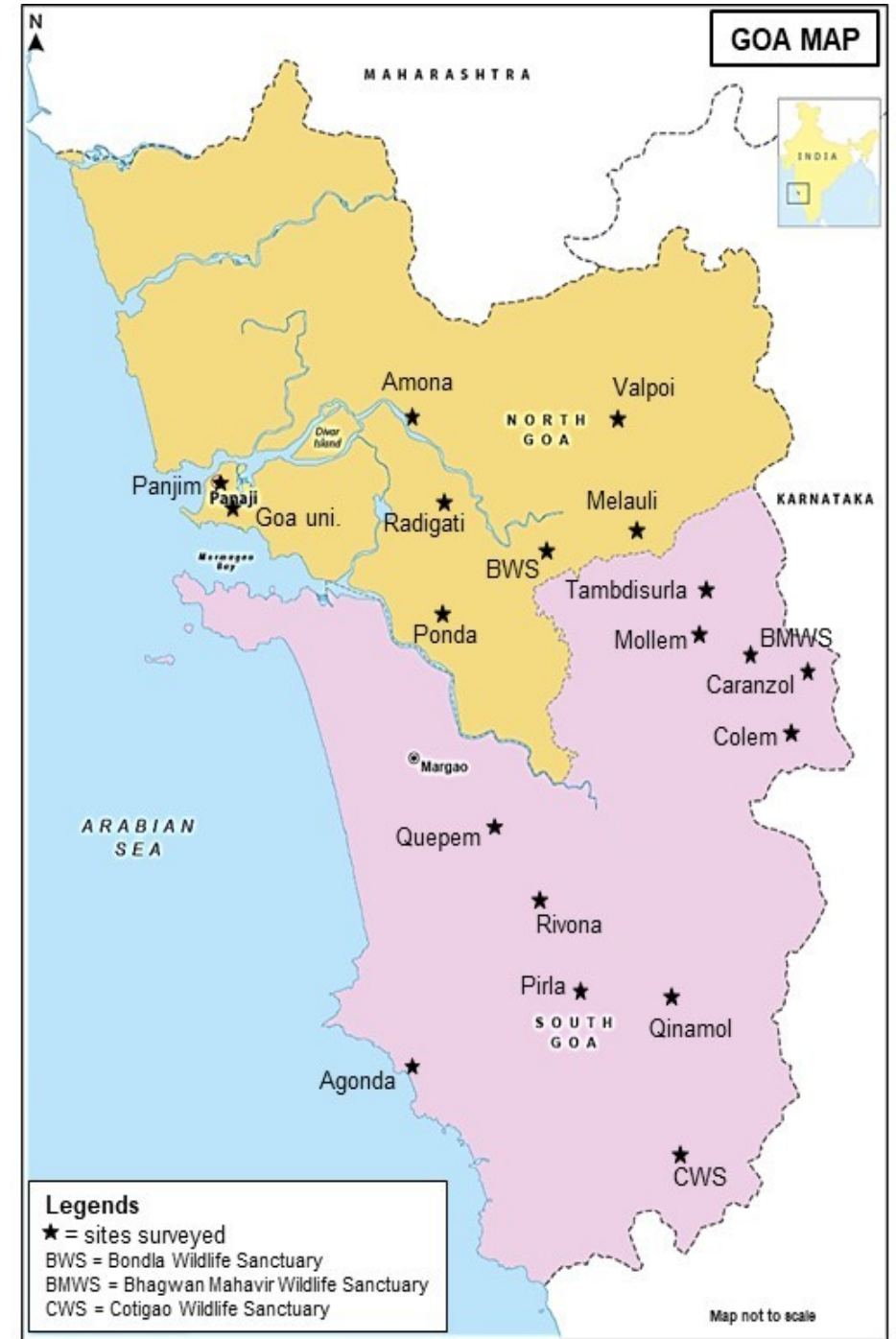

Fig. 1. Map of Goa showing North and South districts and localities surveyed for present study.

$10 \% \mathrm{KOH}$ was used for the detailed study of asci and ascospores. For spot tests, the usual reagents of $\mathrm{K}, \mathrm{C}$ and $\mathrm{P}$ were used. To identify lichens substance, thin layer chromatography (TLC) was performed in solvent system C following the standard method (17). Fresh specimens were preserved in the herbarium of Goa University (GU), and a set of voucher specimens were deposited in herbarium LWG. The specimens were identified up to species level with the help of keys of earlier published literature (18-25). The classification of lichens summarized in reference 26 was followed for arranging species under their respective families. The identity of species was confirmed by matching with type specimens or well-identified specimens available at LWG.

\section{Results}

The study revealed the occurrence of 79 species of pyrenocarpous lichens belonging to 15 genera and seven families in Goa. Five species were recorded for the first time from the country (Table 1). The brief descriptions for the five newly recorded lichens and key for all the pyrenocarpous lichens encountered in Goa are provided. The Pyrenulaceae family was most dominant in the State, with 23 species followed by
Porinaceae (20 spp.) and Monoblastiaceae (13 spp.). Among the genera, Pyrenula was dominant with 24 species, followed by Porina (19 spp.), Anisomeridium (12 spp.) and Strigula (8 spp.). All the species recorded were crustose except for Endocarpon subrosettum A. Singh \& Upreti, which was squamulose. A total of 58 species were found to grow on tree trunks, branches and twigs (corticolous), followed by 16 species that grow on leaves (foliicolous), while five species grow on rocks (saxicolous). Astrothelium meristosporum (Mont. \& Bosch) Aptroot \& Lücking and A. scoria (Fée) Aptroot \& Lücking exhibited their substrate specificity with restricted occurrence on cashew nut (Anacardium occidentale Linn.) trees. The species of Porina and Pyrenula exhibit maximum substrate diversity as they were found growing on various trees, leaves and rocks, while all Strigula species exhibit luxuriant growth only on leaves. Artocarpus integrifolius L.f., Anacardium occidentale L., Cocos nucifera L. and Terminalia elliptica Willd. were the common phorophytes for the growth of pyrenocarpous lichens. Porina interestes (Nyl.) Harm., P. tetracerae (Afz.) Müll. Arg., Pyrenula aspistea (Ach.) Ach., P. oculata A. Singh \& Upreti and Trypethelium eluteriae Spreng. were the most common species of the State. 
Table 1. List of pyrenocarpous lichens recorded from Goa and their distribution [Note: $+=$ present, $-=$ absent, $\mathbf{C R}=$ Crustose, $\mathbf{S Q}=$ Squamulose, $\mathbf{C}=$ Corticolous, $\mathbf{S}=$ Saxicolous, $\mathbf{F}=$ Foliicolous, BMWS $=$ Bhagwan Mahavir Wildlife Sanctuary, $\mathbf{C W S}=$ Cotigao Wildlife Sanctuary, BWS = Bondla Wildlife Sanctuary, Goa Uni. = Goa University, ${ }^{*}$ New record for India]

Sl.

No.

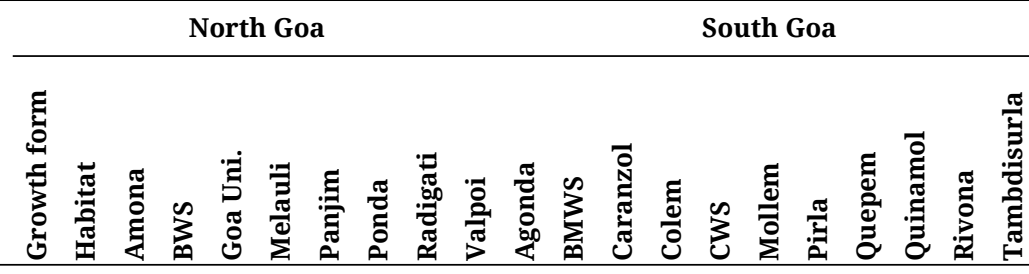

\section{Arthopyreniaceae}

1 Arthopyrenia alboatra (Kremp.) Müll. Arg.

2 A. finkii Zahlbr.

3 A. grisea (Schierch.) Körb.

$4 \quad$ A. indusiata Müll. Arg.

$5 \quad$ A. nidulans Müll. Arg.

\section{Monoblastiaceae}

$6 \quad$ Anisomeridium albidoatrum (Nyl.) R.C. Harris

$7 \quad$ A. angulosum (Müll. Arg.) R.C. Harris

$8 \quad$ A. biforme (Borrer) R.C. Harris

9 A. complanatum (Makhija \& Patw.) R. C. Harris

10 A. consobrinum (Nyl.) Aptroot

11 A. glaucescens (Müll. Arg.) R.C. Harris

12 A. indicum (Makhija \& Patw.) R. C. Harris

13 A. palavanum (Vain.) R.C. Harris

14 A. subnexum (Nyl.) R.C. Harris

15 A. tarmugliense (Makhija \& Patw.) R.C. Harris

16 A. terminatum (Nyl.) R.C. Harris

17 A. ubianum (Vain.) R.C. Harris

18 Monoblastia pellucida Aptroot

\section{Porinaceae}

19 Clathroporina mastoidea (Ach.) R.C. Harris

$20 \quad$ Porina americana Fée

$21 \quad$ P. atlantica (Erich.) P. M. Jørg.

22 P. chrysophora (Stirt.) R. Sant.

23 P. conica R. Sant.

$24 \quad$ P. epiphylla Fée

$25 \quad$ P. exserta Müll. Arg.*

$26 \quad$ P. internigrans (Nyl.) Müll. Arg.

27 P. interestes (Nyl.) Harm.

28 P. karnatakensis Makhija, Adaw. \& Patw.

29 P. nitidula Müll. Arg.

30 P. ochrostoma Makhija, Adaw. \& Patw.

$31 \quad$ P. pallescens R. Sant.

32 P. rufula (Kremp.) Vain.

33 P. siamensis P.M. McCarthy*

34 P. subcutanea Ach.

$35 \quad$ P. subhibernica Upreti

$36 \quad$ P. subinterstes (Nyl.) Müll. Arg.

$37 \quad$ P. tetracerae (Afz.) Müll. Arg.

38 Trichothelium alboatrum Vain.

\begin{tabular}{lllllllllllllllllllllll}
$\mathrm{CR}$ & $\mathrm{C}$ & - & - & - & - & - & - & - & - & - & - & - & - & + & - & - & - & - & - & - \\
\hline $\mathrm{CR}$ & $\mathrm{C}$ & - & - & - & - & - & - & - & - & - & - & - & - & + & - & - & - & - & - & - \\
\hline $\mathrm{CR}$ & $\mathrm{C}$ & - & - & - & - & - & - & - & - & - & - & - & - & + & - & - & - & - & - & - \\
\hline CR & C & - & - & - & - & - & - & - & - & - & + & - & - & + & - & - & - & - & - & + \\
\hline CR & C & - & - & - & - & - & - & - & - & - & - & - & - & - & - & - & - & - & + & -
\end{tabular}

\section{Pyrenulaceae}

39 Lithothelium decumbens (Müll. Arg.) Aptroot

CR C

CR $\mathrm{C}$

CR C

CR C

CR C

CR C

CR C

CR C

CR C

CR $\mathrm{C}$

CR C

CR C

CR C

$40 \quad$ Pyrenula adacta Fée

$41 \quad$ P. aggregata (Fée) Fée

$42 \quad$ P. approximans (Kremp.) Müll. Arg.

$43 \quad$ P. aspistea (Ach.) Ach.

$44 \quad$ P. breutelii (Müll. Arg.) Aptroot

$45 \quad$ P. brunnea Fée

$46 \quad$ P. castanea (Eschw.) Müll. Arg.

$47 \quad$ P. dissimulans (Müll. Arg.) R.C. Harris*

$48 \quad$ P. fetivica (Krempelh.) Müll. Arg.

$49 \quad$. gibberulosa (Vain.) Aptroot

50 P. immissa (Stirt.) Zahlbr.

51 P. leucostoma Ach.

$52 \quad$ P. leucotrypa (Nyl.) Upreti

53 P. mamillana (Ach.) Trevis.

54 P. maravalensis Vain.

$55 \quad$ P. nitidula (Bres.) R.C. Harris.

\begin{tabular}{ll} 
CR & $\mathrm{C}$ \\
\hline $\mathrm{CR}$ & $\mathrm{C}$ \\
\hline $\mathrm{CR}$ & $\mathrm{C}$ \\
\hline $\mathrm{CR}$ & $\mathrm{F}$ \\
$\mathrm{CR}$ & $\mathrm{F}$ \\
\hline $\mathrm{CR}$ & $\mathrm{F}$ \\
$\mathrm{CR}$ & $\mathrm{C}$ \\
$\mathrm{CR}$ & $\mathrm{C}$ \\
$\mathrm{CR}$ & $\mathrm{C}$ \\
$\mathrm{CR}$ & $\mathrm{F}$ \\
$\mathrm{CR}$ & $\mathrm{F}$ \\
\hline $\mathrm{CR}$ & $\mathrm{C}$ \\
$\mathrm{CR}$ & $\mathrm{F}$ \\
\hline $\mathrm{CR}$ & $\mathrm{F}$ \\
$\mathrm{CR}$ & $\mathrm{S}$ \\
\hline $\mathrm{CR}$ & $\mathrm{C}$ \\
$\mathrm{CR}$ & $\mathrm{C}$ \\
$\mathrm{CR}$ & $\mathrm{S}$ \\
$\mathrm{CR}$ & $\mathrm{S}$ \\
$\mathrm{CR}$ & $\mathrm{F}$
\end{tabular}

CR C

CR C

CR C

CR C

CR C

CR C

CR C

CR C

CR C

CR C

CR C

CR C

CR C

CR C

CR C

CR C

CR C 


\begin{tabular}{|c|c|c|c|c|c|c|c|c|c|c|c|c|c|c|c|c|c|c|c|c|c|c|}
\hline 56 & P. ochraceoflava (Nyl.) R. C. Harris & CR & $\mathrm{C}$ & + & - & - & - & - & - & - & + & - & - & - & - & - & - & - & - & - & - & - \\
\hline$\overline{57}$ & P. oculata A. Singh \& Upreti & $\mathrm{CR}$ & $\mathrm{C}$ & - & - & - & + & - & + & - & - & + & + & - & - & - & + & - & - & - & - & - \\
\hline 58 & P. quassiaecola (Fée) Fée & $\mathrm{CR}$ & C & - & - & - & - & - & - & - & - & - & + & - & - & - & - & - & - & - & - & - \\
\hline 59 & P. pyrenastrospora Aptroot* & $\mathrm{CR}$ & $\mathrm{C}$ & - & - & - & - & - & - & - & - & - & - & - & - & + & - & - & - & - & - & - \\
\hline 60 & P. rinodinospora Aptroot* & $\mathrm{CR}$ & $\mathrm{C}$ & - & - & - & - & - & - & - & + & - & - & - & - & - & - & - & - & + & - & - \\
\hline$\overline{61}$ & P. subumbilicata (C. Knight) Aptroot & $\mathrm{CR}$ & $\mathrm{C}$ & - & + & - & - & - & - & - & - & - & + & - & - & - & - & - & - & - & - & - \\
\hline \multicolumn{23}{|c|}{ Strigulaceae } \\
\hline 62 & Strigula antillarum (Fée) Müll. Arg. & CR & $\mathrm{F}$ & - & - & - & - & - & - & - & - & - & - & - & - & + & - & - & - & - & - & - \\
\hline 63 & S. concreta (Fée) R. Sant. & $\mathrm{CR}$ & $\mathrm{F}$ & - & - & - & - & - & - & - & - & - & - & - & - & + & - & - & - & - & - & - \\
\hline$\overline{64}$ & S. janeirensis (Müll. Arg.) Lücking & $\mathrm{CR}$ & $\bar{F}$ & - & - & - & - & - & - & - & - & - & - & - & - & + & - & - & - & - & - & - \\
\hline 65 & S. nitidula Mont. & $\mathrm{CR}$ & $\mathrm{F}$ & - & - & - & - & - & - & - & - & - & - & - & - & + & - & - & - & - & - & - \\
\hline 66 & S. phyllogena (Müll. Arg.) R.C. Harris & CR & $\mathrm{F}$ & - & - & - & - & - & - & - & - & - & - & - & - & + & - & - & - & - & - & - \\
\hline 67 & S. smaragdula Fr. & $\mathrm{CR}$ & $\mathrm{F}$ & - & - & - & - & - & - & - & - & - & + & - & - & + & - & - & - & - & - & - \\
\hline 68 & S. subelegans Vain. & CR & $\mathrm{F}$ & - & - & - & - & - & - & - & - & - & - & - & - & + & - & - & - & - & - & - \\
\hline $69^{*}$ & S. subtilissima (Fée) Müll. Arg. & $\mathrm{CR}$ & $\mathrm{F}$ & - & - & - & - & - & - & - & - & - & - & - & - & + & - & - & - & - & - & - \\
\hline \multicolumn{23}{|c|}{ Trypetheliaceae } \\
\hline$\overline{70}$ & Astrothelium luridum (Zahlbr.) Aptroot \& Lücking & $\mathrm{CR}$ & $\mathrm{C}$ & - & - & - & - & - & - & - & - & - & - & - & & + & - & - & - & - & - & - \\
\hline$\overline{71}$ & A. meristosporum (Mont. \& Bosch) Aptroot \& Lücking & CR & C & - & - & - & - & - & - & - & - & - & - & - & - & + & - & - & - & - & - & - \\
\hline 72 & A. scoria (Fée) Aptroot \& Lücking & $\mathrm{CR}$ & $\mathrm{C}$ & - & - & - & - & - & - & - & - & - & - & - & - & - & - & + & - & - & - & - \\
\hline 73 & $\begin{array}{l}\text { Marcelaria benguelensis (Müll. Arg.) Aptroot, Nelsen } \\
\text { \& Parnmen }\end{array}$ & $\mathrm{CR}$ & C & - & - & - & - & + & - & - & - & - & - & - & - & + & - & - & - & - & - & - \\
\hline$\overline{74}$ & Nigrovothelium bullatum Lücking, Upreti \& Lumbsch & $\mathrm{CR}$ & $\mathrm{C}$ & + & - & - & - & - & - & - & - & - & - & - & - & - & - & - & - & - & - & - \\
\hline 75 & N. tropicum (Ach.) Lücking, M.P. Nelsen \& Aptroot & $\mathrm{CR}$ & C & - & - & - & - & - & - & - & - & - & - & - & - & + & - & - & + & - & - & - \\
\hline 76 & Trypethelium eluteriae Spreng. & CR & $\mathrm{C}$ & - & - & - & - & - & - & - & + & - & - & - & + & + & + & - & + & - & - & - \\
\hline$\overline{77}$ & T. plicatorimosum Mahija \& Patw. & $\mathrm{CR}$ & $\mathrm{C}$ & - & - & - & - & - & - & - & - & - & - & - & - & + & - & - & - & - & - & - \\
\hline \multicolumn{23}{|c|}{ Verrucariaceae } \\
\hline 78 & Endocarpon subrosettum A. Singh \& Upreti & SQ & $\mathrm{S}$ & - & - & + & - & - & - & - & - & - & - & - & - & - & - & - & - & - & - & - \\
\hline$\overline{79}$ & Staurothele fissa (Taylor) Zwackh & $\mathrm{CR}$ & $\mathrm{S}$ & - & - & - & - & + & - & - & - & - & - & - & - & - & - & - & - & - & - & 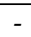 \\
\hline
\end{tabular}

The studied specimens belonged to nineteen localities; eleven were from North Goa, while eight localities were from South Goa. The maximum diversity of pyrenocarpous lichens is recorded in South Goa with 71 species, while North Goa recorded 29 species and 21 species are common between the two districts. It may be noted that South Goa has a more dense forest area which is also fairly well explored compared to North Goa. Among the different localities, Cotigao Wildlife Sanctuary (CWS) is the most extensively surveyed and has maximum diversity of pyrenocarpous lichens with 48 species, while Bhagwan Mahavir Wildlife Sanctuary (BMWS) has 17 and Valpoi has eight species. Ponda and Quinamol localities are poorly represented by pyrenocarpous lichens with one species each. Compared to other sites Ponda and Quinamol are anthropogenically more disturbed which may be the reason for low pyrenocarpous lichen diversity here.

\section{New records}

\section{Porina exserta Müll. Arg. in Flora 71: 548. 1888.}

Thallus corticolous, continuous to rimose, pale brownish to green, smooth to minutely rugulose, 35$80 \mu \mathrm{m}$ thick, ecorticate; prothallus lacking. Perithecial verrucae, hemispherical to subglobose, $0.35-0.96 \mathrm{~mm}$ diam., brownish to blackish, apex rounded; ostiole usually inconspicuous; excipulum 20-30 $\mu \mathrm{m}$ thick, pale orange-brown, centrum $0.2-0.4 \mathrm{~mm}$ wide; subhymenium $15-35 \quad \mu \mathrm{m}$ thick; paraphyses unbranched, 0.7-1.2 $\mu \mathrm{m}$ wide, periphyses absent. Asci elongate to cylindrical $110-1160 \times 14-18 \mu \mathrm{m}$, ascospores elongate to cylindrical, (11-)15-17 (-21) septate, 44-70 × 3.5-5.5 $\mu \mathrm{m}$. Pycnidia absent. (Fig. $2 \mathrm{~A}$ ).

Chemistry:-Thallus K-, C-, KC-, PD-, UV-; no lichen substance present in TLC.
Distribution and ecology:-This species is found growing on the bark of Terminalia paniculata Roth in Cotigao Wildlife Sanctuary between altitude of 100 $200 \mathrm{~m}$. Earlier, this species was reported from Thailand, Christmas Island, the Northern Territory, eastern Queensland, Taiwan and Tahiti (27).

Specimen examined:-INDIA, Goa: South Goa, Cotigao Wildlife Sanctuary, Endrem, N1459'51.2", E 074¹1'52.1", 24 February 2018, P. Randive GU-L 813 (LWG Acc. no. 36250!), on the bark.

Note:-Porina exserta closely resembles $P$. bellendenica Müll. Arg. in having similar perithecial verrucae and ascospores, but $P$. bellendenica differs in having a black basal layer and 15 septate ascospores.

2. Porina siamensis P. M. McCarthy in Lichenologist 31(3): 242. 1999.

Thallus saxicolous, rimose to areolate, smooth, matt, pale greyish to green, $20-80 \mu \mathrm{m}$ thick, ecorticate; prothallus present, grey to black. Perithecia numerous, mostly solitary, semi-immersed to \pm superficial, convex to hemispherical or subconical, 0.2-0.6 mm diam., greenish-brown to black, not overgrown by the thallus; ostiole inconspicuous or in a shallow depression; centrum subglobose to ovate, $0.10-0.25 \mathrm{~mm}$ diam.; excipulum hyaline to pale brown or yellowish-brown, 10-15 $\mu \mathrm{m}$ thick, subhymenium 20-40 $\mu \mathrm{m}$ thick; paraphyses unbranched, $0.8-1 \mu \mathrm{m}$ wide, periphyses absent. Asci cylindrical to elongate, 72-86 × 8-9 $\mu \mathrm{m}$, ascospores 3-septate, cylindrical to fusiform, 14-24 $\times$ 3-4.5 $\mu \mathrm{m}$, lacking perispore. Pycnidia present. (Fig. 2B).

Chemistry:-Thallus K-, C-, KC-, PD-, UV-; no lichen substance present in TLC. 

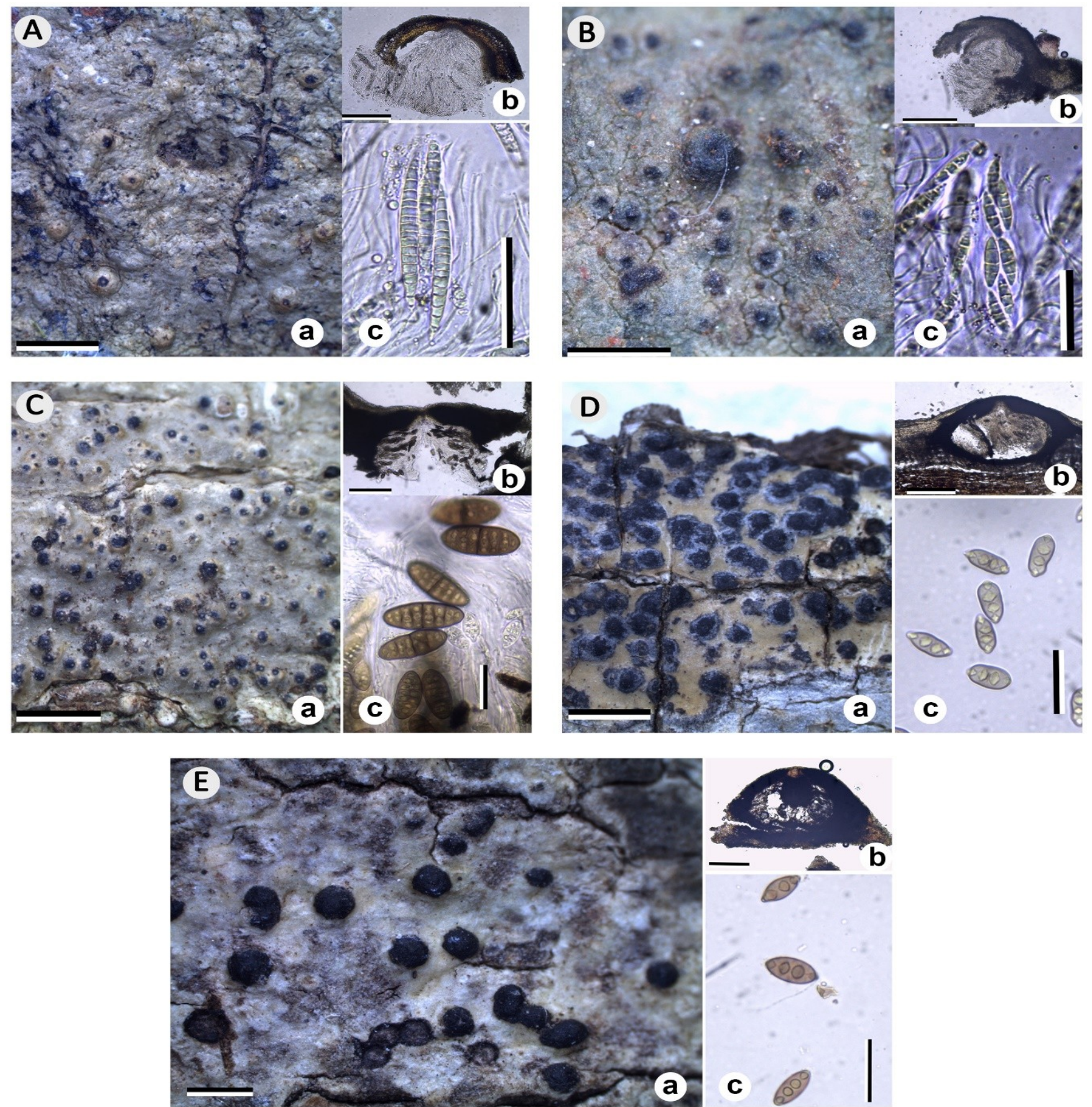

Fig. 2. Habit (a), perithecial section (b) and ascospres (c) of newly recorded pyrenocarpous lichens. A. Porina exserta, B. P. siamensis, C. Pyrenula dissimulans. D. P. pyrenastrospora. E. P. rinodinospora (Scale bars: habit A, C-E $2 \mathrm{~mm}$, B $5 \mathrm{~mm}$; perithecial section A-E $200 \mu$ m; ascospores A. $50 \mu \mathrm{m}, \mathrm{B}-\mathrm{E} 30 \mu \mathrm{m})$.

Distribution and ecology:-Porina siamensis was found growing on an open, exposed rock in Cotigao Wildlife Sanctuary at about $\pm 200 \mathrm{~m}$. Previously this species was known from Thailand (28).

Specimen examined:-INDIA, Goa: South Goa, Cotigao Wildlife Sanctuary, Cuncolim, Agonda, N15¹3'13.0", E07397'99.5", 28 December 2016, P. Randive GU-L434 (LWG Acc. no. 36251!), on rock.

Note:-This species is similar to Porina chlorotica (Ach.) Müll. Arg. and P. fortunata P. M. McCarthy \& Etayo in having black or dark reddish-brown perithecia. $P$. chlorotica also has three septate ascospores, but its perithecia are smaller $(0.2-0.3 \mathrm{~mm}$ diam.) and ascospores are slightly larger (16-32 × 4-6 $\mu \mathrm{m})$. In comparison, $P$. fortunata differs in having seven septate ascospores of size $18-33 \times 5-4 \mu \mathrm{m}$ and perithecia of $0.3-0.4 \mathrm{~mm}$ diam.

3. Pyrenula dissimulans (Müll. Arg.) R.C. Harris in More Florida lichens, Incl. 10 Cent Tour Pyrenol.: 110. 1995.

Thallus corticolous, corticated, smooth, continuous, brownish to yellowish, lacking pseudocyphellae. Perithecia simple, dispersed, conical, emergent, 0.25$0.6 \mathrm{~mm}$ diam., black; ostioles apical black; hamathecium hyaline. Asci cylindrical to clavate, 4-8 spored, ascospores muriform, 25-55 × 14-22 $\mu \mathrm{m}$, 
lumina mostly rounded, at least in the central part of ascospores. Pycnidia not seen. (Fig. 2C).

Chemistry:-Thallus K-, C-, KC-, PD-, UV-; no lichen substance present in TLC.

Distribution and ecology:-This species was found growing on the tree bark of $T$. paniculata in the Goa University campus approximately at an altitude of 100 m. Earlier, this species was known from North and South America (29-31).

Specimens examined:-INDIA, Goa: North Goa, Goa University campus, 02 October 2016, P. Randive, GUL305 (LWG Acc. no. 36252!) on bark, GU- L318 (LWG Acc. no. 36253!) on bark.

Note:- Pyrenula dissimulans is close to P. oleosa R.C. Harris in having corticated thallus, apical ostioles and lacking oil inspersion in hamathecium, but the old ascospores in the latter species are filled with oil.

4. Pyrenula pyrenastrospora Aptroot in Biblthca Lichenol. 64: 165, 1997.

Thallus corticolous, corticated, smooth, brownish, lacking pseudocyphellae. Perithecia mostly aggregated with fused walls, conical, $0.3-0.6 \mathrm{~mm}$ diam.; ostioles apical or when ecentric all opining the same direction, black; hamathecium not inspersed. Asci cylindrical to clavate, 4-8 spored, ascospores brown, 3-septate, fusiform, 16-25 × 6-10 $\mu \mathrm{m}$, lumina in a straight line, terminal lumina directly against the exospores wall. Pycnidia absent. (Fig. 2D).

Chemistry:-Thallus $\mathrm{K}-$, C-, KC-, PD-, UV-; no lichen substance present in TLC.

Distribution and ecology:-This species was found growing on the tree bark of Tectona grandis L.f. in Cotigao Wildlife Sanctuary at altitude $\pm 200 \mathrm{~m}$. Previously this species was known from Papua New Guinea (19).

Specimen examined:-INDIA, Goa: South Goa, Cotigao Wildlife Sanctuary, Bela lake, N14 ${ }^{\circ} 57^{\prime} 23.7^{\prime \prime}$, E07409'09.3", 15 January 2018, P. Randive GU-L655 (LWG Acc. no.36254!), on bark.

Note:-Pyrenula pyrenastrospora is closely related $P$. minarum Vain., which differs in having inspersed hamathecium and partly eccentric ostioles.

5. Pyrenula rinodinospora Aptroot in Lichenologist 44(5): 611-618. 2012.

Thallus corticolous, corticate, smooth, continuous, thin, brownish, without pseudocyphellae. Perithecia simple, dispersed, conical, emergent, 0.3-0.5 mm diam., black, edges without thallus covering; ostioles black, apical; hamathecium hyaline, densely inspersed with oil droplets. Asci cylindrical to clavate, 8-spored, ascospores brown, 3-septate, fusiform, without constrictions, 20-30 × 11-12.5 $\mu \mathrm{m}$, ends mostly pointed, lumina mostly quadrangular, angles blunt, terminal lumina elongated and not separated from the end wall by an endospore layer. Pycnidia not seen. (Fig. 2E).

Chemistry:-Thallus K-, C-, KC-, PD-, UV-; no lichen substance present in TLC.
Distribution and ecology:-This species was found growing on the bark of Ficus benghalensis L. in Coatigao Wildlife Sanctuary and en route to SattariValpoi between altitude 100-200 m. Previously this species was known only from Papua New Guinea (32).

Specimens examined:-INDIA, Goa: South Goa, Cotigao Wildlife Sanctuary, Quinomol, N15'13'130", E074¹1'88.7", 07 December 2016, P. Randive GU-L414 (LWG Acc. no. 36255!) on bark; North Goa, SattariValpoi, N15³1'66.7" E074¹2'41.1", 07 March 2017, P. Randive GU-L 548 (LWG Acc. no. 36256!), on bark.

Note:-Pyrenula rinodinospora closely resembles Pyrenula maravalensis Vain. in having similar morphology, but the latter species differs by the shorter ascospores of 20-25 $\mu \mathrm{m}$ long (18).

\section{Key to the pyrenocarpous lichens of Goa}

1a. Thallus foliicolous ....................................... 2

1b. Thallus otherwise ...................................... 17

2a. Asci functionally unitunicate, entirely thinwalled, perithecia covered by thallus, ascospores transversely 3-9 septate................ 3

2b. Asci functionally bitunicate (fissitunicate), apically thick-walled, perithecia not covered by thallus, ascospores transversely 1-septate..... 10

3a. Perithecia with 5-10 (-12) setae, soft, usually decurved, narrowly acute or bristle-like, whitish or black with white distal halves, ascospores fusiform to narrowly oblong, 7 septate, 25-35 $\times$ 4-6 $\mu \mathrm{m}$ Trichothelium alboatrum

3b. Perithecia lacking setae 4

4a. Area around the ostiole or perithecial surface rough, slightly papillose or tomentose ........... 5

4b. Area around the ostiole and perithecial surface glabrous ................................................. 6

5a. Perithecial wall colourless, ascospores 5 septate, fusiform, 22-30 × 4-5 $\mu \mathrm{m}$... Porina pallescens

5b. Perithecial wall upper part blackish, lower part brownish, ascospores 5 septate, fusiform, 20-32 $\times 4-5.5 \mu \mathrm{m}$ Porina nitidula

6a. Ascospores 3 septate ................................... 7

6b. Ascospores 7-9 septate ................................ 8

7a. Perithecial apex conical, translucent, ascospores $18-27 \times 3-5 \mu \mathrm{m}$ Porina rufula

7b. Perithecia apex rounded, not translucent, ascospores oblong, $14-20 \times 2-4 \quad \mu \mathrm{m}$ Porina chrysophora

8a. Perithecia conical, apex with prominent, short cylindrical extension, ascospores narrowly fusiform to oblong, 34-48 $7 \times 4-6 \mu \mathrm{m}$ Porina conica

8b. Perithecia apex without any extension ........... 9

9a. Perithecia conical to wart-shaped when mature, with black dot around ostiole, ascospores narrowly fusiform, 33-63 × 4-6 $\mu \mathrm{m}$, white prothallus sometimes present 
9b. Perithecia lens shaped to hemispherical, without dark spot, prothallus absent, ascospores oblong, 26-33 × 3-4 $\mu \mathrm{m}$....... Porina epiphylla

10a. Ascospores large, 35-70 × 4-8 $\mu \mathrm{m}$, often breaking into halves (each cell often with up to 3 secondary septa), oblong, with distinct constriction at septum ....Strigula janeirensis

10b. Ascospores small, up to $25 \mu \mathrm{m}$ long .............. 11

11a. Distal cell of the ascospore enlarged............. 12

11b. Distal cell not enlarged .............................. 13

12a. Thallus subcuticular, bright green, ascospores irregularly biseriate, $15-25 \times 4-6 \mu \mathrm{m}$

Strigula antillarum

12b. Thallus epiphyllous, pale greenish to bluish grey, ascospores biseriate, 15-25 × 4-6 $\mu \mathrm{m}$

Strigula subelegans

13a. Ascospores breaking into halves either inside or outside the asci 14

13b. Ascospores not breaking 15

14a. Thallus thin (8-15 $\mu \mathrm{m})$, bright metallic green, margin effuse, with a thin blackish line, ascospores 8-12 × 2-3 $\mu \mathrm{m}$....Strigula nitidula

14b. Thallus thick (15-30 $\mu \mathrm{m})$, pale greyish green, margin crenulate to lobulate, lacking blackish line, ascospores 8-12 × 2-3 $\mu \mathrm{m}$

Strigula concreta

15a. Thallus elobate, photobiont cells rectangular to angular-rounded, perithecia pure black, conical, delimited from surrounding thallus, ascospores 9-12 × 2-3 $\mu \mathrm{m}$ Strigula phyllogena

15b. Thallus crenulate lobate to distinctly lobate . 16

16a. Thallus with distinct lobes leaving small to large interspaces, greenish-brown, perithecia completely exposed but covered by thin thallus layer, ascospores oblong acicullar, with a slight constriction at septum, 10-18 × 2-3 $\mu \mathrm{m}$..............

\section{.Strigula subtilissima}

16b. Thallus entire to crenulate or lobulate, sometimes whole thallus lobate-laciniate, bright green, perithecia immersed to erumpent, covered by algiferous thallus tissue up to ostiolum, ascospores 14-24 × 4-6 $\mu \mathrm{m}$

Strigula smaragdula

17a. Thallus saxicolous 18

17b. Thallus corticolous 22

18a. Thallus squmulose, squmules imbricate, up to $2.5 \mathrm{~mm}$ wide, perithecia 1-6 per squamule, ascospores 2 per asci, brown, muriform, 30-40 $\times$ 12-14 $\mu \mathrm{m}$ Endocarpon subrosettum

18b. Thallus crustose, continuous to areolate ..... 19

19a. Ascospores muriform, 2 per acus, brown, 70-90 $\times 15-25 \mu \mathrm{m}$, perithecia sunken, algal cell in the hymenium globose, thallus rimose areolate, greyish brown Staurothele fissa

19b. Ascospores transversely septate, hyaline .... 20 20a. Ascospores 3 septate, cylindrical to fusiform, 14$24 \times 3-5 \mu \mathrm{m}$, perithecia $0.2-0.6 \mathrm{~mm}$ diam., greenish-brown to black, not overgrown by the thallus, ostiole inconspicuous or in a shallow depression

Porina siamensis

20b. Ascospores 7-9 septate 21

21a. Ostiole conspicuous, periostiolar region brown to black, perithecia $0.5-0.8 \mathrm{~mm}$ diam., 7-9 septate, $23-34 \times 5-9 \mu \mathrm{m}$, fusiform with rounded ends

Porina subinterstes

21b. Ostiole usually inconspicuous, periostiolar area pale to dark brown or blackish, perithecia 0.3$0.8 \mathrm{~mm}$ diam., ascospores 7 septate, $24-44 \times 4-7$ $\mu \mathrm{m}$, cylindrical or narrowly obclavate.

\section{Porina tetracerae}

22a. Ascospores hyaline .23

22b. Ascospores greyish brown to brown ............ 57

23a. Ascospores simple, subglobose to ellipsoid, surface ornamented with cristae, 9-13 × 7-9 $\mu \mathrm{m}$, thallus slightly squamulose

Monoblastia pellucida

23b. Ascospores septate .24

24a. Ascospores transversely septate .................. 25

24b. Ascospores muriform ................................ 56

25a. Ascospores 1-septate ................................ 26

25b. Ascospores 3 or more septate....................... 41

26a. Perithecial wall hyphal, containing bark cells, pseudoparaphyses branched, but not anastomosing ........................................... 27

26b. Perithecial wall cellular, lacking bark cells, pseudoparaphyses slender, branched and anastomosing especially above the asci ....... 30

27a. Ascospores 2 per asci, 1-septate, sometimes constricted at both ends and appearing as 3 septate, large $(40-60 \times 15-25 \mu \mathrm{m})$

Arthopyrenia finkii

27b. Ascospores 8 per asci 28

28a. Distal cell of the ascospore is larger, ascospore 30-45 × 14-18 $\mu \mathrm{m}$, perithecia solitary Arthopyrenia nidulans

28b. Both the cells are almost equal in size ......... 29

29a. Ostiole mammilate, perithecia solitary to 2-3 aggregate, ascospores 25-43 × 6-11 $\mu \mathrm{m}$.

Arthopyrenia indusiata

29b. Ostiole not mammilate, perithecia solitary, ascospores 10-18 × 5-7 $\mu \mathrm{m}$.....

Arthopyrenia alboatra

30a. Thallus UV+ ............................................. 31

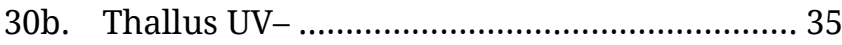

31a. Distal cell of the ascospores is larger, ascospores 23-30 × 7-10 $\mu \mathrm{m}$, perithecia single, ostiole eccentric

Anisomeridium ubianum

31b. Both the cells are of almost equal size .......... 32 
32a. Ostiole eccentric, perithecia single, ascospores 27-35 × 8-10 $\mu \mathrm{m}$. Anisomeridium angulosum

32b. Ostiole apical 33

33a. Ascospores smaller, 14-19 × 4-65 $\mu \mathrm{m}$, narrowly fusiform, ascus mostly biseriate. Anisomeridium albidoatrum

33b. Ascospores $>20 \mu \mathrm{m}$ long .... 34

34a. Ascospores 25-30 × 9-13 $\mu \mathrm{m}$, elliptical to broadly elliptical, ascus mostly uniseriate.... Anisomeridium consobrinum

34b. Ascospores 30-45 × 13-16 $\mu \mathrm{m}$, ovoid to broadly ovoid, ends somewhat pointed Anisomeridium indicum

35a. Ascospores 2-4 per ascus, 40-66 × 10-17 $\mu \mathrm{m}$, perithecia solitary, ostiole black with white wide pore........Anisomeridium tarmugliense

35b. Ascospores 8 per ascus ................................ 36

36a. Distal cell of ascospore larger in size ............ 37

36b. Both the cells of ascospores are of almost equal in size 38

37a. Ostiole eccentric, ascospores 19-30 × 7-12 $\mu \mathrm{m}$ Anisomeridium palavanum

37b. Ostiole apical, ascospores 16-21 × 4-7 $\mu \mathrm{m}$ Anisomeridium biforme

38a. Ostiole apical, ascospores 23-27 × 9-11 $\mu$, fusiform, biseriate or irregularly arranged in ascus Anisomeridum subnexum

38b. Ostiole eccentric .39

39a. Ascospores granular ornamented, fusiform to narrowly ovoid, 19-30 × 6-8 $\mu \mathrm{m}$ Anisomeridium terminatum

39b. Ascospores smooth .40

40a. Ascospores fusiform, with pointed ends, 30-37 $\times$ 9-11 $\mu \mathrm{m}$ Anisomeridium glaucescens

40b. Ascospores 30-40 × 6-8 $\mu \mathrm{m}$ Anisomeridium complanatum

41a. Ascospores lumina diamond-shaped 42

41b. Ascospores lumina rectangular-shaped .45

42a. Perithecia in distinct or diffuse pseudostroma, laterally covered by thallus 43

42b. Perithecia solitary to crowded, fully exposed, black 44

43a. Perithecia in diffuse stroma, upper portion blackish brown with whitish rim, erumpent, 0.3$0.5 \mathrm{~mm}$ diam., hamathecium inspersed, ascospores 3 septate, 15-27 × 7-10 $\mu \mathrm{m}$, fusiformellipsoid

Astrothelium scoria

43b. Perithecia in distinct stroma, upper portion dark, erumpent to prominent, 0.8-1.5 mm broad, ostiolar region internally with yellow pigment, hamathecium clear, ascospores 7-11 septate, fusiform, 55-88 × 13-28 $\mu \mathrm{m}$. 44a. Thallus strongly verrucose-bullate, olive-green to brownish, perithecia $0.2-0.3 \mathrm{~mm}$ diam., sessile, stioles visible as tiny black dots, ascospores fusiform, 20-25 × 8-10 $\mu \mathrm{m}$

.Nigrovothelium bullatum

44b. Thallus smooth to uneven, olive-green to yellowish-brown, perithecia $0.2-0.3 \mathrm{~mm}$ diam., prominent to sessile, subglobose to barrelshaped with a flattened top, ostiolar area greyish, ascospores fusiform-ellipsoid, 20-25 × 7-10 $\mu \mathrm{m}$

Nigrovothelium tropicum

45a. Ascospore 3 septate, perithecia solitary or 2-3 aggregated, ostiole apical, umbonate, 19-23 × 5$7 \mu \mathrm{m}$, thallus endophloeodal.

Arthopyrenia grisea

45b. Ascospores more than 3 septate 46

46a. Perithecia highly crowded to pseudostromatic ............................................................. 47

46b. Perithecia solitary or aggregated, never in pseudostroma, but in verrucae 48

47a. Perithecia in diffuse pseudostroma, brownblack, $0.5-1.0 \mathrm{~mm}$, basally covered by thallus but upper part exposed, flattened and discshaped, ascospores 12-17-septate, fusiform, 45$55 \times 6-8 \mu \mathrm{m}$, thallus olive-green to yellowish Trypethelium plicatorimosum

47b. Perithecia in distinct pseudostroma, brownish to dark brown, covered by yellow pigment, 1-2 $\mathrm{mm}$ diam., ascospores 9-13-septate, fusiform, 37-52 × 8-11 $\mu \mathrm{m}$.

Trypethelium eluteriae

48a. Ostiole $\mathrm{K}+$ reddish, perithecia 0.45-0.6 $\mathrm{mm}$ diam., ascospores 7 septate, elliptic, 19-30 × 4-7 $\mu \mathrm{m}$ Clathroporina mastoidea

48b. Ostiole K- ................................................... 49

49a. Ascospores $>13$ septa ................................ 50

49b. Ascospores 6-13 septa ...............................51

50a. Perithecia brownish blackish, ostiole inconspicuous, ascospores 11-21 septa, elongate to cylindrical, 47-70 × 4-6 $\mu \mathrm{m}$.

Porina exserta

50b. Perithecia greenish grey, area around ostiole black, ascospores 13-16 septate, straight to curved with pointed ends, 50-70 × 5-7 $\mu \mathrm{m}$ Porina subhibernica

51a. Hamathecium inspersed with oil ....................................................... 52

51b. Hamathecium not inspersed ........................................................................ 53

52a. Ascospores 7-9 septate, oblong ellipsoid, 60-85 × 12-20 $\mu \mathrm{m}$, ostiolar region brown black. Porina subcutanea

52b. Ascospores 11-12 septate, fusiform, 89-92 × 16$20 \mu \mathrm{m}$, ostiolar region black

Porina americana

53a. Ostiolar region yellow to light brown, surrounded by black priostiolar rim, ascospores 
7-9 septate, fusiform with acute ends, 43-50 × 4$6 \mu \mathrm{m}$ Porina ochrostoma

53b. Ostiolar region variously coloured, peri ostiolar rim lacking 54

54a. Ostiolar region brown-black, ascospores 7-8 septate, fusiform, acute ends, $32-48 \times 4-7 \mu \mathrm{m} . . .$.

\section{Porina interestes}

54b. Ostiolar region otherwise, ascospores much larger 55

55a. Ostiolar region orange, pinkish, reddish to brownish, rarely dark brown, ascospores 7-9 (13) septate, fusiform with rounded ends, 44-80 $\times$ 7-13 $\mu \mathrm{m}$

Porina atlantica

55b. Ostiolar region orange-brown to black to black, ascospores fusform round ended, 45-60 ×11-18 $\mu \mathrm{m}$ Porina internigrans

56a. Thallus olive-green, yellow-orange pruinose, UV+, perithecia flattened top, solitary to irregularly grouped and confluent, covered with yellow-orange pigment, hamathecium densely inspersed, ascospores fusiform-ellipsoid, 50-80 $\times 17-23 \mu \mathrm{m}$ Marcelaria benguelensis

56b. Thallus light olive-green, pigmented pruina absent, UV+, flattened top, solitary to grouped, covered by thallus up to ostiole, hamathecium inspersed, ascospores oblong-fusiform, 120-220 $\times 25-40 \mu \mathrm{m}$, with thickened median septum........

Astrothelium meristosporum

57a. Ascospores transversely 3 septate ................ 58

57b. Ascospores submuriform to muriform ......... 74

58a. Perithecia in pseudostroma, 1-12 carpic, thallus yellowish-brown to brown, $\mathrm{K}+\mathrm{red}$, ascospores ellipsoidal, $13-23 \times 6-10 \mu \mathrm{m}$

\section{Pyrenula leucotrypa}

58b. Perithecia solitary or aggregated, not forming pseudostroma 59

59a. Ascomata mostly aggregated, with fused walls but with separate ostioles, conical, 0.3-0.6 mm diam., ostioles apical, black, ascospores brown, 3 septate, fusiform 16-25 × 6-10 $\mu \mathrm{m}$.

\section{Pyrenula pyrenastrospora}

59b. Ascomata solitary 60

60a. Ascospores red-brown, lumina becoming rounded when older, 15-20 × 9-10 $\mu \mathrm{m}$, ellipsoid, hamathecium inspersed, thallus olive-brown shining

Lithothelium decumbens

60b. Ascospores grey to brown, rarely red-brown and then lumina angular 61

61a. Ostiole eccentric, perithecia solitary, ascospores 35-45 × 15-18 $\mu \mathrm{m}$, terminal lumina separated from the exospore wall by endospore thickening Pyrenula adacta

61b. Ostiole apical 62

62a. Hamathecium inspersed 63

62b. Hamathecium not inspersed. .69 63a. Thallus pseudocyphellate, yellow-brown buff, perithecia solitary or 1-4 coalescing, ostiole indistinct, ascospores oblong ellipsoid, 32-45(60) $\times 15-27 \mu \mathrm{m}$

Pyrenula immissa

63b. Thallus lacking pseuodcyphellae 64

64a. Ascospore 27-50 × 15-18 $\mu \mathrm{m}$, oblong ellipsoid, perithecia solitary, completely embedded in thalline verrucae, ostiole indistinct, thallus ochre-yellowish brown ...... Pyrenula oculata

64b. Ascospore $<30 \mu \mathrm{m}$ long .65

65a. End lumina of the ascospore is elongated ....66

$65 \mathrm{~b}$. All lumina \pm rounded to angular 67

66a. Thallus brownish to pale brown, ascomata emergent 0.3-0.5 $\mathrm{mm}$ diam., hemithecium densely impressed with oil droplets, ascospores 20-25 × 6-10 $\mu \mathrm{m}$....... Pyrenula maravalensis

66b. Thallus ochraceous yellow to brownish, ascomata semiemergent $0.5-1.0 \mathrm{~mm}$ diam., hemithecium without oil droplets, ascospores 20-30 × 11-13 $\mu \mathrm{m}$.... Pyrenula rinodinospora

67a. Ostiole mamillate papillate, dull black, perithecia solitary, ascospores oblong ellipsoid, 17-20×7-9 $\mu \mathrm{m}$, thallus buff to yellow-brown .....

Pyrenula mamillan

67b. Ostiole otherwise, centrum I \pm perithecia solitary or semi-solitary, ascospores ellipsoid, thallus brownish yellow to brown ................ 68

68a. Psudocyphellae present, ascocarp 0.8-1.0 $\mathrm{mm}$ in diameter, conical- hemispherical, centrum I+ blue, ascospores 16-24 × 8-13 $\mu \mathrm{m}$

Pyrenula fetivica

68b. Psudocyphellae absent, ascocarp 1.0-2.5 (-3.0) $\mathrm{mm}$ in diameter, flat, convex to conicodepressed, centrum I -, ascospores 15-17 × 5-7 $\mu \mathrm{m}$

Pyrenula castanea

69a. Thallus with pseudocyphellae, brownish to olive-green, ascospore fusiform, 22-38 × 8-15 $\mu \mathrm{m}$, lumina rounded to somewhat angular Pyrenula quassiaecola

69b. Thallus lacking pseudocyphellae .................. 70

70a. Terminal lumina mostly separated from exospore wall .......................................... 71

70b. Terminal lumina not separated from exospore wall 73

71a. Perithecia slightly larger, 0.6-1.0 mm diam., ascospores oblong ellipsoid, 12-17 × 5-7 $\mu \mathrm{m}$.......

.Pyrenula brunnea

71b. Perithecia smaller ........................................ 72

72a. Perithecia 0.2-0.6 mm diam., ascospores oblong ellipsoid, 17-22 × 9-10 $\mu \mathrm{m}$...Pyrenula aspistea

72b. Perithecia 0.4-0.7 mm diam., ascospores oblong ellipsoid, $15-21 \times 8-10 \mu \mathrm{m}$

Pyrenula aggregata

73a. End lumina elongated, ascospore 20-29 $\times 9-11$ $\mu \mathrm{m}$ Pyrenula approximans 
73b. All lumina \pm rounded to angular, ascospores 13 $16 \times 7-8 \mu \mathrm{m}$ Pyrenula nitidula

74a. Ostioles eccentric, fused, perithecia usually in groups of 2-6, ascospores submuriform, with 5 transverse septa and few longitudinal septa, fusiform, with pointed ends, 24-32 × 10-13 $\mu \mathrm{m}$, thallus yellowish to olive-green

\section{Pyrenula subumbilicata}

74b. Ostiole apical, not fused, perithecia mostly solitary 75

75a. Thallus and perithecia yellow to orange, indistinctly to faintly papillate, ascospores ellipsoidal, $12-18 \times 7-10 \mu \mathrm{m}$

\section{Pyrenula ochraceoflava}

75b. Thallus and perithecia otherwise .76

76a. Old ascospores filled with orange oil, muriform, 25-30 × 10-15 $\mu \mathrm{m}$, pantropical distribution ...................................... Pyrenula breutelii

76b. Old ascospores lacking orange oil, submuriform................................................ 77

77a. Thallus yellow-brown, pseudocyphellate, UV+ yellowish, ostioles indistinct to mamillatepapillate, hamethecium not inspresed, ascospores 23-35 × 10-12 $\mu \mathrm{m}$ long

\section{Pyrenula gibberulosa}

77b. Thallus otherwise, ostioles apical, ascospores larger, up to $45-55 \mu \mathrm{m}$ long .......................... 78

78a. Lumina of the ascospore mostly round, ascospore 25-55 × 14-22 $\mu$, ostioles apical, naked, black, thallus yellowishbrown........................Pyrenula dissimulans

78b. Lumina relatively large and angular, ascospore 24-46 × 9-20 $\mu \mathrm{m}$, ostiolar region naked, brownish-black to black, thallus greenish straw coloured, yellow to yellow-brown

Pyrenula leucostoma

\section{Discussion}

The semi-evergreen forest in the Goa region exhibits maximum diversity of pyrenocarpous species. The trees along the streams in moist, shady habitats bear luxuriant growth of pyrenolichens which mostly belong to Porina and Pyrenula species. The occurrence of pyrenocarpous lichens indicates the abundant presence of smooth-barked trees in the State. Further, the occurrence of a large number of foliicolous lichens indicates that the State has several healthy and undisturbed forests. Bhagwan Mahavir Wildlife Sanctuary as a whole and within Cotigao Wildlife Sanctuary, Avem, Bela to Zombolim,, Endrem to Tulshimol, Kuske waterfall area, Marlem, Nadkem and Ravan Donger are few such pristine habitats. With the addition of five new records, the total number of lichens species in Goa State increases to 165 species, out of which pyrenocarpous lichens represent almost half (48\%).

\section{Conclusion}

The present study indicates the occurrence of a rich diversity of pyrenocarpous lichens in the Western
Ghats forests of Goa. It can be noted that among the protected areas within Goa, Cotigao Wildlife Sanctuary represents more number of pyrenocarpous lichens. Further, along with the forest areas within Goa, the coconut, arecanut orchards and several cultivated plants also provide suitable habitats for lichen growth. The present study will be highly useful for monographic studies on pyrenocarpous lichens of India or the world and for environmental monitoring studies in the area.

\section{Acknowledgements}

We are thankful to Director, CSIR-NBRI, Lucknow, for providing the laboratory facilities for the research, to Publication Ethics Committee of CSIR-NBRI for carefully scrutinizing the manuscript and approving it for publication with ms number MS/2020/08/03. Authors PR and MKJ are thankful to Goa University for financial assistance under the Ph.D. programme and to the Forest Department of Goa for permission to survey Cotigao Wildlife Sanctuary. PR is also thankful to DST for financial assistance under Women Scientist Scheme (SR/WOS-A/LS-354/2016). GKM is thankful to SERB for financial assistance under the NPDF scheme (PDF/2017/000356).

\section{Authors' contributions}

PR collected fresh samples of pyrenocarpous lichens from Goa and carried out their preliminary identification. GKM studied pyrenocarpous lichen specimens preserved in herbarium LWG and drafted the manuscript. SN authenticated identity of some pyrenocaprous lichens reported in the manuscript, improved the manuscript and supervised the whole study. DKU authenticated identity of some pyrenocarpous lichen specimens and suggested improvement in the manuscript. MKJ conceptualized the idea of studying lichens of Goa, supervised the work of author PR and provided intellectual inputs to the study.

\section{Conflict of interests}

The authors do not have any conflict of interests to declare.

\section{References}

1. Randive P, Nayaka S, Janarthanam MK. An updated checklist of lichens from Goa with new records from Cotigao Wildlife Sanctuary. Cryptogam Biodiversity and Assessment 2017;2(1):26-36. https://doi.org/10.21756/cab.v2i01.8609

2. Randive R, Joseph S, Nayaka S, Janarthanam MK. Notes on foliicolous lichens from Western Ghats part of Goa, India. Indian Journal of Forestry. 2017;40(3):217-21.

3. Randive P, Nayaka S, Janarthanam MK. Lichens of Cotigao Wildlife Sanctuary, Goa. International Journal of Life Sciences, Special Issue, 2018;A9:31-36

4. Randive P, Joseph S, Gupta P, Nayaka S, Janarthanam MK. Additional records of the follicolous lichens to the State of Goa. Indian Forester. 2019;145(7):687-88.

5. Joseph S, Nayaka S, Randive P, Upreti DK. New records and a key to the species of Malmidea (lichenized Ascomycota) from In- 
dia. Feddes Repertorium. 2018;129(3):189-92. https://doi.org/ 10.1002/fedr.201800011

6. Gupta P, Randive P, Nayaka S, Daimari R, Joseph S, Janarthanam MK. New records of graphidioid and thelotremoid lichens from India. Mycotaxon. 2020;135:345-54. https://doi.org/ $10.5248 / 135.345$

7. Aptroot A. Tropical pyrenocarpous lichens. A phylogenetic approach. In: Galloway DJ, editor. Tropical lichens: Their systematics, conservation and ecology. Systematics Association Special Volume. Oxford; Clarendon Press; 1991. p. 253-73.

8. Singh A. Two new species of Anthracothecium from India. Indian Journal of Biological Research. 1985;1(1):82-85.

9. Upreti DK. Lichen genus Pyrenula in India: I Pyrenula subducta spore type. J. Hattori Botanical Laboratory. 1990;68:269-78.

10. Upreti DK. Lichen genus Pyrenula from India: The species with spores of Pyrenula brunnea type. Bull Soc Bot France, Lett Bot. 1991;138(3):241-47.

11. Upreti DK. Lichen genus Pyrenula from India: IV. Pyrenula cayennensis spore type. Cryptog. Bryol Lichénol. 1991;12(1):41-46.

12. Upreti DK. Lichen genus Pyrenula from India: VII. Pyrenula mas tophora spore type. Feddes Repert. 1992;103(3-4):279-96. https://doi.org/10.1002/fedr.19921030315

13. Upreti DK. Lichen genus Pyrenula from India: II. Pyrenula camptospora spore type, III. Pyrenula pinguis spore type. Acta Bot Gallica. 1993;140(5):519-23. $\quad$ https://doi.org/ 10.1080/12538078.1993.10515628

14. Upreti DK, Pant G. Notes on Arthopyrenia species from India. Bryologist. 1993;96(2):226-32. https://doi.org/10.2307/3243807

15. Phatak S, Nayaka S, Upreti DK, Singh SM, Samuel C. Preliminary observation on lichen flora of Cotigao Wildlife Sanctuary, Goa, India. Phytotaxonomy. 2004;4:104-06.

16. Nayaka S, Upreti DK, Phatak S, Samuel C. Lichens of Bondla and Bhagwan Mahavir Wildlife Sanctuaries, Goa. Biological Memoirs. 2004;30(2):115-19.

17. Orange AP, James W, White FJ. Microchemical methods for the identification of lichens. U.K: British Lichen Society; 2001.

18. Awasthi DD. A Key to the Microlichens of India, Nepal and Sri Lanka. Bibliotheca Lichenologica. 1991;40:1-340.

19. Aptroot A. A world key to the species of Anthracothecium and Pyrenula. Lichenologist. 2012;44(1):5-53. https://doi.org/10.1017/ s0024282911000624

20. Aptroot A, Ertz D, Salazar JAE, Gueidan C, Diaz JAM, Schumm F, Weerakoon G. Forty-six new species of Trypetheliaceae from the tropics. Lichenologist. 2016;48(6):609-38. https://doi.org/ $10.1017 / \mathrm{s} 002428291600013 \mathrm{x}$

21. Aptroot A, Cáceres MEDS. New Trypetheliaceae from the Amazon basin in Rondônia (Brazil), the centre of diversity of the genus Astrothelium. Lichenologist. 2016;48(6):693-712. https:// doi.org/10.1017/s0024282915000584

22. Aptroot A, Lücking R A revisionary synopsis of the Trypetheliaceae (Ascomycota: Trypetheliales). Lichenologist. 2016;48(6):763-982. https://doi.org/10.1017/S0024282916000487
23. Aptroot A. Three new species of Lithothelium (Pyrenulaceae) from China and Thailand, with a revised world key and annotated list of species. Lichenologist. 2006;38(6):541-48. https://doi.org/10.1017/s0024282906005913

24. Upreti DK. Notes on corticolous and saxicolous species of Porina with Porina subhibernica sp. nov. Bryologist. 1994;97(1):73-79. https://doi.org/10.2307/3243353

25. Upreti DK. A key to the lichen genus Pyrenula from India with nomenclatural notes. Nova Hedwigia. 1998;66(3-4):111-30.

26. Lücking R, Hodkinson BP, Leavitt SD. The 2016 classification of lichenized fungi in the Ascomycota and Basidiomycota - Approaching one thousand genera. Bryologist. 2017;119(4):361416. https://doi.org/10.1639/0007-2745-119.4.361

27. McCarthy PM. Additional lichens records from Australia 68 . Tropical prenolichens. Australian Lichenology. 2008;63:10-16.

28. McCarthy PM. Three new species of Porina (Trichotheliaceae) from Thailand. Lichenologist. 1999;31(3):239-46. https://doi.org/10.1006/lich.1998.0187

29. Harris RC. More Florida Lichens. Including the 10® Tour of the Pyrenolichens. Bronx (New York): Publ. by the Author; 1995.

30. Käffer MI, Koch NM, Aptroot A, De Martins SMA. New records of corticolous lichens for South America and Brazil. Plant Ecology and Evolution. 2015;148(1):111-18. https://doi.org/10.5091/plecevo.2015.961

31. Seavey F, Seavey J. Four new species and sixteen new lichen records for North America from Everglades National Park. Bryologist. 2014;117(4):395-404. https://doi.org/10.1639/00072745-117.4.395

32. Aptroot A, Schumm F, Cáceres MES. Six new species of Pyrenula from the tropics. Lichenologist. 2012;44(5):611-18. https:// doi.org/10.1017/S0024282912000254

\section{Additional information}

Peer review information: Plant Science Today thanks Sectional Editor and the other anonymous reviewers for their contribution to the peer review of this work.

Reprints and permissions information is available at https://horizonepublishing.com/journals/index.php/PST/open_access_policy

Publisher's Note: Horizon e-Publishing Group remains neutral with regard to jurisdictional claims in published maps and institutional affiliations.

To cite this article: Randive P, Mishra G K, Nayaka S, Upreti D K, Janarthanam M K. Pyrenocarpous lichens in Goa with five new records to India. Plant Science Today. 2021;8(4):889-899. https://doi.org/10.14719/pst.2021.8.4.1265

Plant Science Today, published by Horizon e-Publishing Group, is covered by Scopus, Web of Science, BIOSIS Previews, Clarivate Analytics, etc. See https://horizonepublishing.com/journals/index.php/PST/indexing_abstracting 